\title{
Application of Method of Collocation on Lines for Solving Nonlinear Hyperbolic Problems
}

\author{
By E. N. Houstis*
}

\begin{abstract}
A collocation on lines procedure based on piecewise polynomials is applied to initial/boundary value problems for nonlinear hyperbolic partial differential equations. Optimal order a priori estimates are obtained for the error of approximation. The Crank-Nicholson discretization in time is studied and convergence rates of the collocation-Crank-Nicholson procedure are established. Finally, the superconvergence is verified at particular points for linear hyperbolic problems.
\end{abstract}

Introduction. We consider the nonlinear hyperbolic problem

$$
p(x, t, u) D_{t}^{2} u-q(x, t, u) D_{x}^{2} u=f\left(x, t, u, D_{x} u\right), \quad(x, t) \in(0,1) \times(0, T],
$$

subject to the initial conditions

$$
u(x, 0)=u_{0}, \quad D_{t} u(x, 0)=u_{1}, \quad x \in(0,1),
$$

and to Dirichlet boundary conditions for $t>0$. We examine the convergence of the collocation on lines procedure using piecewise polynomials with continuous first derivatives as the approximating functions.

In Section 4 we obtain optimal-order asymptotic estimates for the error of the approximation in the $L_{\infty}$-norm. In Section 5, the Crank-Nicholson discretization of the resulting system of ordinary differential equations is studied and convergence rates of the collocation on lines-Crank-Nicholson procedure are established. Finally, in Section 6 the superconvergence phenomenon is established locally for a linear hyperbolic problem.

The method of collocation on lines was proposed first by Kantorovich [7]. The convergence of this method for a problem of mathematical physics was investigated by E. B. Karpilovskaya [8] . Yartsev [11] , [10] proved convergence for linear elliptic and biharmonic type problems using trigonometric polynomials as basis functions. Douglas and Dupont [3], have studied the same method using piecewise cubic Hermite polynomials for a nonlinear parabolic problem and in [4] verified the superconvergence locally for the heat equation. Finally, Douglas and Dupont [5] generalized and extended their results in [3], [4]. The results in this paper are from the author's thesis [6].

1. Preliminary Results. Let $\Delta_{x}=\left(x_{i}\right)_{0}^{N}$ be a partition of $[0,1], I=[0,1]$, $h_{j} \equiv\left|x_{j+1}-x_{j}\right|, I_{j} \equiv\left[x_{j}, x_{j+1}\right]$ and $h \equiv \max _{j}\left|x_{j+1}-x_{j}\right|$. Throughout this paper 
we denote by $\mathbf{P}_{r}$ the set of polynomials of degree less than $r$ and $\mathbf{P}_{r, \Delta_{x}}$ the set of functions that are polynomials of degree $r-1$ in each subinterval $\left[x_{i}, x_{i+1}\right]$. We take $-1<\rho_{1}<\rho_{2}<\cdots<\rho_{k}<1$ and $w_{j}>0, j=1, \ldots, k$, to be Gaussian points and weights, respectively, so that

$$
\int_{-1}^{+1} p(x) d x=\sum_{i=1}^{k} p\left(\rho_{i}\right) w_{i}, \quad p \in \mathbf{P}_{2 k}([-1,1]) .
$$

The Gaussian points and weights in the subinterval $\left[x_{j}, x_{j+1}\right]$ are

$$
\xi_{k j+i} \equiv\left(x_{j}+x_{j+1}\right) / 2+\rho_{i} h_{j} / 2, \quad w_{i}^{*}=h_{j} w_{i} / 2, \quad i=1, \ldots, k .
$$

We introduce two pseudo-inner products corresponding to Gaussian quadrature and composite Gaussian quadrature:

$$
(f, g)_{h_{j}} \equiv \frac{h_{j}}{2} \sum_{i=1}^{k} w_{i} f\left(\xi_{k j+i}\right) \cdot g\left(\xi_{k j+i}\right)
$$

and

$$
(f, g)_{h} \equiv \sum_{j=0}^{N-1}(f, g)_{h_{j}}
$$

with

$$
|f|_{h} \equiv \sum_{j=0}^{N-1}(f, f)_{h_{j}}
$$

For later use, we state without proof the lemmas:

LEMмA 1.1. The seminorm $|f|_{h}$ is positive definite for all $f \in \mathbf{P}_{k+2, \Delta_{x}} \cap C^{1}[0,1]$ with $f(0)=f(1)=0$.

Lemma 1.2. If $f, g \in \mathbf{P}_{k+2, \Delta_{x}} \cap C^{1}[0,1]$, then

$$
-\left(D_{x}^{2} f, g\right)_{h}=\left(D_{x} f, D_{x} g\right)-\left.D_{x} f \cdot g\right|_{0} ^{1}
$$

$$
+\frac{(k+1) k}{(2 k) !} \sum_{j} \frac{D_{x}^{k+1} f_{j}}{(k+1) !} \cdot \frac{D_{x}^{k+1} g_{j}}{(k+1) !} \int_{x_{j}}^{x_{j+1}} \prod_{i=1}^{k}\left(x-\xi_{k j+i}\right)^{2} d x .
$$

Lemma 1.3. If $f \in\left\{v \in \mathbf{P}_{k+2, \Delta_{x}} \cap C^{1}, v(0)=v(1)=0\right\}$, then

and

$$
\left(D_{x} f, D_{x} f\right) \leqslant-\left(D_{x}^{2} f, f\right)_{h} \leqslant 2\left(D_{x} f, D_{x} f\right)
$$

LEMma 1.4. If $f \in \mathbf{P}_{k+2, \Delta_{x}} \cap C^{1}[0,1]$, then

$$
\left|D_{x} f\right|_{h}^{2} \leqslant\left(D_{x} f, D_{x} f\right)
$$

where $\lambda$ is the maximum eigenvalue of the matrix $A_{k+1} \equiv\left[\Sigma_{l=1}^{k} w_{l} L_{i}\left(\rho_{l}\right) L_{j}\left(\rho_{l}\right)\right]$ and $L_{i}$ denotes the ith degree Legendre polynomials in $[-1,1]$.

Let $H^{k}$ be the Sobolev space of functions having $L^{2}$-derivatives of order $k$ on $I$ and $H_{0}^{k} \equiv\left\{u \in H^{k} \mid u(0)=u(1)=0\right\}$. 
LEMmA 1.5. For $f \in H^{1}$ we have

$$
\left(D_{x} f, D_{x} f\right)+|f|_{h}^{2} \geqslant 1 / 4\|f\|_{H^{1}(I)}^{2} .
$$

The above lemmas are established in [6], proofs also appear in [5]. Lemmas 1.2, 1.3 and 1.5 have been first proved for the case of cubic Hermite polynomials by Douglas and Dupont [3].

2. Approximation Theory. In [6] we show that $R_{k}(x) \equiv D_{x}^{k}\left(1-x^{2}\right)^{k+2}, k=$ $0,1, \ldots$, on $(-1,1)$ are orthogonal polynomials. By Rodriques' formula we see that $D_{x}^{2} R_{k}(x)=D_{x}^{k+2}\left(1-x^{2}\right)^{k+2}$ is a multiple of the Legendre polynomial on the interval $(-1,1)$. We now establish some properties of these polynomials.

LEMMA 2.1. If $k \geqslant 3$,

$$
\left(D_{x}^{\mu} R_{k-2}, x^{\nu}\right)_{h}=0, \quad \mu=0,1,2, \nu \leqslant \mu .
$$

PROof. Since $D_{x}^{\mu} R_{k-2} x^{\nu}$ is a polynomial of degree $K+2-\mu+\nu$, we have for $k \geqslant 3$,

$$
\left(D_{x}^{\mu} R_{k-2}, x^{\nu}\right)_{h}=\int_{-1}^{1} D_{x}^{\nu} R_{k-2} x^{\nu} d x
$$

Lemma 2.1 now follows by using integration by parts and the fact that $D_{x}^{\mu} R_{k-2}$ vanishes at $x= \pm 1$ and $D_{x}^{2} R_{k-2}$ vanishes at the Gaussian points. Note that for $k \geqslant 2$,

$$
\left(D_{x} R_{k-2}, 1\right)_{h}=\left(D_{x}^{2} R_{k-2}, x^{\nu}\right)_{h}=0 .
$$

We define an interpolation operator

$$
T_{h}: C^{1}(I) \rightarrow \mathbf{P}_{k+2, \Delta_{x}} \cap C^{1}(I)
$$

such that

$$
\begin{aligned}
\left(T_{h} v\right)\left(x_{l}\right) & =v\left(x_{l}\right), \\
\left(D_{x} T_{h} v\right)\left(x_{l}\right) & =\left(D_{x} v\right)\left(x_{l}\right), \quad l=0,1, \ldots, N, \\
\left(T_{h} v\right)\left(\tau_{i, j}\right) & =v\left(\tau_{i, j}\right), \quad i=1, \ldots, k, j=1, \ldots, N,
\end{aligned}
$$

where $\tau_{i, j} \equiv x_{j}+\sigma_{i}\left(x_{j+1}-x_{j}\right)$ and the $\sigma_{i}$ 's are the roots in the interval $(0,1)$ of the orthogonal polynomials $R_{k-2}(x)$.

LemmA 2.2. Assume that $u \in H^{k+4}(I)$ and let $e \equiv u-T_{h} u$. Then there is a constant $K$ independent of $h$ so that

$$
\begin{aligned}
&\left|D_{x}^{l} e\right|_{h} \leqslant K h^{k-l+2}\|u\|_{H^{k+2}(I)}, \quad l=0,1, \\
&\left|D_{x}^{2} e\right|_{h} \leqslant K h^{k-l+1}\|u\|_{H^{k+3}(I)}, \\
&\left|\left(D_{x} e, 1\right)_{h}\right| \leqslant K h^{2 k+5 / 2}\|u\|_{H^{k+3}(I)}, \\
&\left|\left(D_{x}^{2} e, 1\right)_{h}\right| \leqslant K h^{2 k+5 / 2}\|u\|_{H^{k+4}(I)} .
\end{aligned}
$$

Proof. It follows easily from Lemma 2.1 and Peano's Kernel Theorem [9]. 
3. Collocation on Lines. In this section we consider the problem of approximating the solution of the nonlinear hyperbolic equation

$$
p(x, t, u) D_{t}^{2} u-q(x, t, u) D_{x}^{2} u=f\left(x, t, u, D_{x} u\right), \quad(x, t) \in(0,1) \times(0, T],
$$

subject to the initial conditions

$$
u(x, 0)=\alpha_{1}(x), \quad D_{t}(x, 0)=\alpha_{2}(x), \quad 0<x<1,
$$

and the boundary conditions

$$
u(0, t)=0, \quad u(1, t)=0, \quad 0<t \leqslant T .
$$

Assume that the coefficients satisfy

$$
0<c_{1} \leqslant p(x, t, u) \leqslant C_{1}, \quad c_{2} \leqslant q(x, t, u) \leqslant C_{2},
$$

for $0 \leqslant x \leqslant 1,0 \leqslant t \leqslant T$ and $-\infty<u<+\infty$. Also, we assume that $p, q, f$ are continuously differentiable functions of their arguments and uniformly bounded.

Throughout, we assume that this problem has a solution, $u$.

Let $S_{\Delta_{x}} \equiv \mathrm{P}_{k+2, \Delta_{x}} \cap C^{1}$ and $S_{\Delta_{x}} \cap H_{0}^{1}$ be spanned by the basis functions $\left\{B_{i}\right\}_{1}^{k N}$. We seek an approximation $u_{h}(x, t)$ to $u$ of the form

$$
u_{h}(x, t)=\sum_{i=1}^{k N} \beta_{i}(t) B_{i}(x) .
$$

The coefficients $\left\{\beta_{i}(t)\right\}_{i=1}^{k N}$ as functions of time are the solutions of the nonlinear ordinary differential equations

$$
\begin{aligned}
\left\{p\left(u_{h}\right) D_{t}^{2} u_{h}-q\left(u_{h}\right) D_{x}^{2} u_{h}-f\left(u_{h}, D_{x} u_{h}\right)\right\}\left(\xi_{i}, t\right) & =0, \\
0 & <t \leqslant T, i=1, \ldots, k N,
\end{aligned}
$$

and

$$
u_{h}\left(\xi_{i}, 0\right)=\hat{\alpha}_{1}\left(\xi_{i}\right), \quad D_{t} u_{h}\left(\xi_{i}, 0\right)=\hat{\alpha}_{2}\left(\xi_{i}\right), \quad k=1, \ldots, k N,
$$

where $\hat{\alpha}_{1}, \hat{\alpha}_{2}$ are the $S_{\Delta_{x}}$-interpolants of $\alpha_{1}(x), \alpha_{2}(x)$ respectively.

Although these are the equations which one solves in practice, the analysis is more conveniently made if one considers the equivalent problem of finding $u_{h} \in S_{\Delta_{x}} \cap H_{0}^{1}$ such that

$$
\begin{aligned}
\left(p\left(u_{h}\right) D_{t}^{2} u_{h}-q\left(u_{h}\right) D_{x}^{2} u_{h}-f\left(u_{h}, D_{x} u_{h}\right), B_{i}\right)_{h} & =0 \\
0 & <t \leqslant T, i=1, \ldots, k N,
\end{aligned}
$$

and

$$
u_{h}\left(\xi_{i}, 0\right)=\hat{\alpha}_{1}\left(\xi_{i}\right), \quad D_{t} u_{h}\left(\xi_{i}, 0\right)=\hat{\alpha}_{2}\left(\xi_{i}\right), \quad i=1, \ldots, k N .
$$

Lemma 3.1. The collocation method (3.5), (3.6) and the discrete Galerkin method (3.7), (3.8) each possess a unique solution for $0<t \leqslant T$. Moreover, these solutions are identical if the processes are started from the same initial values.

PROoF. It follows from Lemma 4.1 in [5]. 
4. Error Analysis. In this section, we find a priori error bounds for the collocation on lines procedure. We consider the problem of finding $u_{h} \in S_{\Delta_{x}} \cap H_{0}^{1}$ such that

$$
\left(p\left(u_{h}\right) D_{t}^{2} u_{h}-D_{x}^{2} u_{h}-f\left(u_{h}, D_{x} u_{h}\right), v\right)_{h}=0, \quad 0<t \leqslant T,
$$

for all $v \in S_{\Delta_{x}} \cap H_{0}^{1}$.

In order to find estimates for the error $u-u_{h}$ in the $L_{\infty}$-norm, we assume that $u(\cdot, t) \in C^{1}(I)$ and define $w(\cdot, t) \equiv T_{h} u$ which is in $S_{\Delta_{x}}$. Then we find a priori bounds for the difference $w-u_{h} \in S_{\Delta_{x}}$; and applying known approximation results to the difference $u-w$, we obtain bounds for the error of the collocation on lines procedure.

If $X$ is a normed space and $\psi:[0, T] \rightarrow X$, define

$$
\|\psi\|_{L^{2}(0, T ; X)}=\int_{0}^{T}\|\psi(t)\|_{X}^{2} d t, \quad\|\psi\|_{L^{\infty}(0, T ; X)}=\sup _{0 \leqslant t \leqslant T}\|\psi(t)\|_{X} .
$$

THEOREM 4.1. If

(i) the coefficients in (3.1) have bounded third derivatives and satisfy conditions (3.4),

(ii) $u \in L^{\infty}\left(0, T ; H^{k+4}\right), D_{t} u \in L^{2}\left(0, T ; H^{k+4}\right)$ and $D_{t}^{2} u \in L^{2}\left(0, T ; H^{k+4}\right)$,

(iii) $u_{h}(x, 0), D_{t} u_{h}(x, 0)$ are the $S_{\Delta_{x}}$ interpolants of $u(x, 0)$ and $D_{t} u(x, 0)$, respectively, then for the error of approximation we have

$$
\begin{aligned}
\left\|u-u_{h}\right\|_{L^{\infty}\left(0, T ; L^{\infty}\right)} \leqslant K\left[\|u\|_{L^{\infty}\left(0, T ; H^{k+4}(I)\right)}\right. & +\left\|D_{t} u\right\|_{L^{2}\left(0, T ; H^{k+4}(I)\right)} \\
& \left.+\left\|D_{t}^{2} u\right\|_{L^{2}\left(0, T ; H^{k+4}(I)\right)}\right] h^{k+2},
\end{aligned}
$$

where $K$ is a constant independent of $h$ and $u$.

Proof. Let $\eta \equiv u-w$ and $\zeta \equiv w-u_{h}$. Then (3.1), (4.1) imply that

$$
\begin{aligned}
\left(p\left(u_{h}\right) D_{t}^{2} \zeta-D_{x}^{2} \zeta, v\right)_{h}= & \left(-p_{u}^{1} \zeta D_{t}^{2} w-p(w) D_{t}^{2} \eta-p_{u}^{2} \eta D_{x}^{2} u, v\right)_{h} \\
& +\left(D_{x}^{2} \eta, v\right)_{h}+\left(\left[f\left(w, D_{x} u\right)-f\left(w, D_{x} w\right)\right], v\right)_{h} \\
& +\left(f_{u}^{1} \eta+f_{u}^{2} \zeta+f_{D_{x} u}^{3} D_{x} \zeta, v\right)_{h} .
\end{aligned}
$$

In (4.2) we choose $v=D_{t} \xi$ and in [6] we show that

$$
\begin{aligned}
& 1 / 2\left[\left|\sqrt{p\left(u_{h}\right)} D_{t} \zeta\right|_{h}^{2}+|\zeta|_{h}^{2}-\left(D_{x}^{2} \zeta, \zeta\right)_{h}\right] \\
& \leqslant K \int_{0}^{t}\left\{|\zeta|_{h}^{2}+\left|D_{x} \zeta\right|_{h}^{2}\right\} d \tau+\int_{0}^{t}\left\{|\eta|_{h}^{2}+\left|D_{t}^{2} \eta\right|_{h}^{2}\right\} d \tau+K \int_{0}^{t}\left|D_{t} \zeta\right|_{h}^{2} \\
& +K\left\{|\zeta|_{h}^{2}(0)-\left(D_{x}^{2} \zeta, \zeta\right)_{h}(0)+\left|\sqrt{p\left(u_{h}\right)} D_{t} \zeta\right|_{h}^{2}(0)\right\} \\
& +\int_{0}^{t}\left(D_{x}^{2} \eta, D_{t} \zeta\right)_{h} d \tau+\int_{0}^{t}\left(f\left(w, D_{x} u\right)-f\left(w, D_{x} w\right), D_{t} \zeta\right)_{h} d \tau \text {. }
\end{aligned}
$$

Integration by parts gives

$$
\int_{0}^{t}\left(D_{x}^{2} \eta, D_{t} \zeta\right)_{h} d \tau=\left.\left(D_{x}^{2} \eta, \zeta\right)_{h}\right|_{0} ^{t}-\int_{0}^{t}\left(D_{t} D_{x}^{2} \eta, \zeta\right)_{h} d \tau
$$


and

$$
\begin{aligned}
\int_{0}^{t}( & \left.f\left(w, D_{x} u\right)-f\left(w, D_{x} w\right), D_{t} \zeta\right)_{h} d \tau \\
& =\left.\left(f\left(w, D_{x} u\right)-f\left(w, D_{x} w\right), \zeta\right)_{h}\right|_{0} ^{t}-\int_{0}^{t}\left(D_{t}\left\{f\left(w, D_{x} u\right)-f\left(w, D_{x} w\right)\right\}, \zeta\right)_{h} d \tau
\end{aligned}
$$

Using Poincare's inequality, the elementary inequality $|c d| \leqslant(1 / 4 p) c^{2}+p d^{2}$ and Lemma 2.2 in [6] we have obtained

$$
\begin{aligned}
\left|\int_{0}^{t}\left(D_{t} D_{x}^{2} \eta, \zeta\right)_{h} d \tau\right| \leqslant & \frac{1}{16} \int_{0}^{t}\left[-\left(D_{x}^{2} \zeta, \zeta\right)_{h}+|\zeta|_{h}^{2}\right] d \tau \\
& +K \sum_{i=1}^{N} h_{j}^{2 k+4} \int_{0}^{t}\left\|D_{t} u(\cdot, \tau)\right\|_{H^{k+4}\left(I_{j}\right)}^{2} d \tau .
\end{aligned}
$$

Using Taylor's theorem, we can easily show that

$$
\begin{aligned}
\left(f\left(w, D_{x} u\right)-f\left(w, D_{x} w\right), w-u_{h}\right)_{h} & \\
& =\sum_{j=1}^{N}\left(\left.f_{D_{x} u}\left(w, D_{x} w\right)\right|_{x=\xi_{k(j-1)+1}} D_{x} \eta+\omega h_{j} D_{x} \eta, \zeta\right)_{h_{j}},
\end{aligned}
$$

where $\omega$ is bounded independent of $h_{j}$. It follows from Lemma 2.2

$$
\begin{aligned}
\mid\left(\left.f_{D_{x} u}\right|_{x=}\right. & \left.\xi_{k(j-1)+1} D_{x} \eta, \zeta\right)_{h_{j}} \mid \\
& \leqslant K h_{j}^{k+2}\left[\|u(\cdot, t)\|_{H^{k+3}\left(I_{j}\right)}|\zeta|_{h_{j}}+\|u(\cdot, t)\|_{H^{k+3}\left(I_{j}\right)}\left\|D_{x} \zeta(\cdot, t)\right\|_{L^{2}\left(I_{j}\right)}\right],
\end{aligned}
$$

and

$$
\left|\left(\omega h_{j} D_{x} \eta, \zeta\right)_{h_{j}}\right| \leqslant K h_{j}^{k+2}\|u(\cdot, t)\|_{H^{k+2}\left(I_{j}\right)}|\zeta|_{h_{j}} .
$$

Moreover, we obtain

$$
\begin{aligned}
& \left|\left(f\left(w, D_{x} u\right)-f\left(w, D_{x} w\right), \zeta\right)_{h}\right| \\
& \quad \leqslant \frac{1}{16}\left[-\left(D_{x}^{2} \zeta, \zeta\right)_{h}+|\zeta|_{h}^{2}\right]+K \sum_{j=1}^{N} h_{j}^{2 k+4}\|u(\cdot, t)\|_{H^{k+3}\left(I_{j}\right)}^{2}
\end{aligned}
$$

Following similar arguments as above, we show that

$$
\begin{aligned}
& \int_{0}^{t}\left(D_{t}\left\{f\left(w, D_{x} u\right)-f\left(w, D_{x} w\right)\right\}, \zeta\right)_{h} d \tau \\
& \leqslant \frac{1}{16} \int_{0}^{t}\left[-\left(D_{x}^{2} \zeta, \zeta\right)_{h}+|\zeta|_{h}^{2}\right] d \tau \\
&+K \sum_{j=1}^{N} h_{j}^{2 k+4} \int_{0}^{t}\left[\|u(\cdot, \tau)\|_{H^{k+3}\left(I_{j}\right)}^{2}+\left\|D_{t} u(\cdot, \tau)\right\|_{H^{k+3}\left(I_{j}\right)}^{2}\right] d \tau
\end{aligned}
$$

It follows from (4.3)-(4.5), (1.3) and Gronwall's Lemma [6] that 


$$
\begin{aligned}
& \left\|w-u_{h}\right\|_{L^{\infty}\left(0, T ; L^{\infty}\right)}^{2} \\
& \leqslant K\left[\left\|\left(w-u_{h}\right)(\cdot, 0)\right\|_{H^{1}(I)}^{2}+\left\|D_{t}\left(w-u_{h}\right)(\cdot, 0)\right\|_{L^{2}(I)}^{2}\right] \\
& +\sum_{j=1}^{N} h_{j}^{2 k+4}\left\{\|u\|_{L^{\infty}\left(0, T ; H^{k+4}\left(I_{j}\right)\right)}^{2}\right. \\
& \left.+\left\|D_{t} u\right\|_{L^{2}\left(0, T ; H^{k+4}\left(I_{j}\right)\right)}^{2}+\left\|D_{t}^{2} u\right\|_{L^{2}\left(0, T ; H^{k+4}\left(I_{j}\right)\right)}^{2}\right\}
\end{aligned}
$$

It is an elementary consequence of Peano's Kernel Theorem that

$$
\|u-w\|_{L^{\infty}\left(0, T ; L^{\infty}\right)}^{2} \leqslant K \sum_{j=1}^{N} h_{j}^{2 k+4}\|u\|_{L^{\infty}\left(0, T ; H^{k+4}\left(I_{j}\right)\right)}^{2} .
$$

Finally, from (4.6), (4.7) and assumption (iii) it follows that

$$
\begin{aligned}
& \left\|u-u_{h}\right\|_{L^{\infty}\left(L^{\infty}(I)\right)} \\
& \quad \leqslant K h^{k+2}\left[\|u\|_{L^{\infty}\left(H^{k+4}(I)\right)}+\left\|D_{t} u\right\|_{L^{2}\left(H^{k+4}(I)\right)}+\left\|D_{t}^{2} u\right\|_{L^{2}\left(H^{k+4}(I)\right)}\right] .
\end{aligned}
$$

This concludes the proof of Theorem 4.1.

5. Computational Considerations. In this section, we discuss the question of actually solving the system of ordinary differential equations (3.5), (3.6).

Let

$$
\begin{gathered}
u_{h}^{j} \equiv u_{h}^{j}(x)=u_{h}^{j}\left(x, t^{j}\right), \quad t^{j} \equiv j \Delta t, \quad \Delta t=T / N, \\
v^{j+1 / 2} \equiv\left(v^{j+1}+v^{j}\right) / 2, \quad v^{j, 1 / 4} \equiv \frac{1}{4} v^{j+1}+\frac{1}{2} v^{j}+\frac{1}{4} v^{j-1}, \\
\partial_{t} v^{j+1 / 2} \equiv\left(v^{j+1}-v^{j}\right) / \Delta t, \quad \partial_{t}^{2} v^{j} \equiv\left(v^{j+1}-2 v^{j}+v^{j-1}\right) /(\Delta t)^{2} .
\end{gathered}
$$

Then the Crank-Nicholson-Collocation approximation $\left\{u_{h}^{j}\right\}_{0}^{N}$ is defined such that

(i) $\left\{p\left(t^{j}, u_{h}^{j, 1 / 4}\right) \partial_{t}^{2} u_{h}^{j}-q\left(t^{j}, u_{h}^{j, 1 / 4}\right) D_{x}^{2} u_{h}^{j, 1 / 4}-f\left(t^{j}, u_{h}^{j, 1 / 4}, D_{x} u_{h}^{j, 1 / 4}\right)\right\}\left(\xi_{i}\right)=0$,

$$
i=1, \ldots, k N, j=0, \ldots, N-1,
$$

(ii) $u_{h}^{j}(0)=u_{h}^{j}(1)=0, \quad j=0, \ldots, N$.

At the end of this section we discuss the choice of $u_{h}^{0}, u_{h}^{1}$. In order to analyze the convergence of the solution of (5.2) we consider the equivalent to (5.2) normalized problem

$$
\left(p\left(t^{j}, u_{h}^{j, 1 / 4}\right) \partial_{t}^{2} u_{h}^{j}, v\right)_{h}-\left(D_{x}^{2} u_{h}^{j, 1 / 4}, v\right)_{h}=\left(f\left(t^{j}, u_{h}^{j, 1 / 4}, D_{x} u_{h}^{j, 1 / 4}\right), v\right)_{h}
$$

$$
v \in S_{\Delta_{x}} \cap H_{0}^{1}, 0 \leqslant j<N
$$

Also, we introduce the notation

$$
\begin{aligned}
\|u\|_{L_{\Delta t}^{2}(0, T ; X)}^{2} & \equiv \sum_{0<t^{j}<T}\left\|u^{j}\right\|_{X}^{2} \Delta t, \\
\|u\|_{L_{\Delta t}^{\infty}(0, T ; X)}^{2} & \equiv \max _{0<t^{j}<T}\left\|u^{j}\right\|_{X}^{2},
\end{aligned}
$$




$$
\|u\|_{\tilde{L}_{\Delta t}^{2}(0, T ; X)}^{2} \equiv \sum_{0<t^{j}<T}\left\|u^{j}\right\|_{X}^{2} \Delta t
$$

THEOREM 5.1. Assume the hypotheses (i), (ii) of Theorem 4.1 hold. Further, assume $D_{t}^{3} u, D_{t}^{4}, u$ are in $L^{\infty}\left(0, T ; L^{2}(I)\right)$ and

$$
\left\|\left(u_{h}-w\right)^{1 / 2}\right\|_{H^{1}(I)}+\left\|\partial_{t}\left(u_{h}-w\right)^{1 / 2}\right\|_{L^{2}(I)}=O\left(h^{k+2}\right) .
$$

For $\Delta t$ sufficiently small there exists a unique solution of the Crank-NicholsonCollocation equations (5.2) and for the error of approximation we have

$$
\left\|u-u_{h}\right\|_{L_{\Delta t}^{\infty}\left(0, T ; L^{\infty}\right)} \leqslant C\left(h^{k+2}+(\Delta t)^{2}\right),
$$

where $C$ depends on $u$ and is independent of $h, \Delta t$.

Proof. It is easily seen that a unique solution of (5.2) exists under assumption (i) and (3.3) for $\Delta t$ sufficiently small. Throughout this proof we use the notation $w \equiv$ $T_{h} u, \eta \equiv u-w$ and $\zeta \equiv u_{h}-w$. First, we observe that $u$ satisfies

$$
\left(p\left(u^{j, 1 / 4}\right) \partial_{t}^{2} u^{j}\right)_{h}-\left(D_{x}^{2} u^{j, 1 / 4}, v\right)_{h}=\left(f\left(u^{j, 1 / 4}, D_{x} u^{j, 1 / 4}\right), v\right)_{h}+\left(e^{j}, v\right)_{h}
$$

for $v \in S_{\Delta_{x}} \cap H_{0}^{1}$, where $\left\|e^{j}\right\|_{L^{2}(I)}=O\left(\Delta t^{2}\right)\left\|D_{t}^{4} u\right\|_{L^{2}(I)}$.

After straightforward calculations and the application of the Mean Value Theorem, we obtain

$$
\begin{aligned}
\left(p\left(u_{h}^{j, 1 / 4}\right) \partial_{t}^{2} \zeta^{j}, v\right)_{h}-\left(D_{x}^{2} \zeta^{j}, v\right) & \\
= & \left(p^{*} \xi^{j, 1 / 4} \partial_{t}^{2} w^{j}, v\right)_{h}+\left(p\left(w^{j, 1 / 4}\right) \partial_{t}^{2} \eta^{j}, v\right)_{h} \\
& +\left(p^{* *} \eta^{j, 1 / 4} \partial_{t}^{2} u^{j}, v\right)_{h}+\left(e^{j}, v\right)_{h}-\left(D_{x}^{2} \eta^{j, 1 / 4}, v\right)_{h} \\
& +\left(f_{u}^{*} \eta^{j, 1 / 4}+f_{u}^{* *} \zeta^{j, 1 / 4}+f_{D_{x}}^{*} D_{x} \zeta^{j, 1 / 4}, v\right)_{h} \\
& +\left(f\left(w^{j, 1 / 4}, D_{x} w^{j, 1 / 4}\right)-f\left(w^{j, 1 / 4}, D_{x} u^{j, 1 / 4}\right), v\right)_{h} .
\end{aligned}
$$

In (5.5), we choose as test function $v=\left(\zeta^{j+1}-\zeta^{j-1}\right) / 2 t$ and then we obtain

$$
\begin{aligned}
& \frac{1}{2 \Delta t}\left\{\left[\left.\left|\sqrt{\left.p\left(u_{h}^{j, 1 / 4}\right) \partial_{t} \zeta^{j+1 / 2}\right|_{h} ^{2}}+\right| \zeta^{j+1 / 2}\right|_{h} ^{2}-\left(D_{x}^{2} \zeta^{j+1 / 2}, \zeta^{j+1 / 2}\right)_{h}\right]\right. \\
& \left.-\left[\left|\sqrt{p\left(u^{j, 1 / 4}\right)} \partial_{t} \zeta^{j-1 / 2}\right|_{h}^{2}+\left|\zeta^{j-1 / 2}\right|_{h}^{2}-\left(D_{x}^{2} \zeta^{j-1 / 2}, \zeta^{j-1 / 2}\right)_{h}\right]\right\} \\
& \leqslant C\left[\left|\zeta^{j+1 / 2}\right|_{h}^{2}+\left|\zeta^{j-1 / 2}\right|_{h}^{2}+\left|\partial_{t}^{2} \eta^{j}\right|_{h}^{2}+\left|\eta^{j, 1 / 4}\right|_{h}^{2}+\left|\partial_{t} \zeta^{j+1 / 2}\right|_{h}^{2}\right. \\
& \left.+\left|\partial_{t} \zeta^{j-1 / 2}\right|_{h}^{2}+\left|D_{x} \zeta^{j, 1 / 4}\right|_{h}^{2}+\left|e^{j}\right|_{h}^{2}\right] \\
& +\left|\left(D_{x}^{2} \eta^{j, 1 / 4}, \frac{\zeta^{j+1}-\zeta^{j-1}}{2 \Delta t}\right)_{h}\right| \\
& +\left|\left(f\left(w^{j, 1 / 4}, D_{x} w^{j, 1 / 4}\right)-f\left(w^{j, 1 / 4}, D_{x} u^{j, 1 / 4}\right), \frac{\zeta^{j+1}-\zeta^{j-1}}{2 \Delta t}\right)_{h}\right|
\end{aligned}
$$

where $C$ is a generic constant. 
Following the same arguments as in Section 4 and using Lemma 2.2, we get

$$
\begin{aligned}
\Delta t & \sum_{j=1}^{n-1}\left(D_{x}^{2} \eta^{j, 1 / 4}, \frac{\zeta^{j+1}-\zeta^{j-1}}{2 \Delta t}\right)_{h} \\
\leqslant & \frac{1}{\epsilon}\left\{-\left(D_{x}^{2} \zeta^{n-1 / 2}, \zeta^{n-1 / 2}\right)_{h}+\left|\zeta^{n-1 / 2}\right|_{h}^{2}-\left(D_{x}^{2} \zeta^{1 / 2}, \zeta^{1 / 2}\right)_{h}+\left|\zeta^{1 / 2}\right|_{h}^{2}\right\} \\
& +K \max _{0 \leqslant t^{j} \leqslant T} \sum_{i=1}^{N-1} h_{i}^{2 k+4}\left\|u^{j}\right\|_{H^{k+4}\left(I_{i}\right)}+\max _{0 \leqslant t^{j} \leqslant T}\left\|e_{1}^{j}\right\|_{L^{2}(I)}^{2}
\end{aligned}
$$

$$
\begin{aligned}
+\frac{1}{2 \epsilon} \Delta t \sum_{j=1}^{n-1}\left\{-\left(D_{x}^{2} \zeta^{j+1 / 2}, \zeta^{j+1 / 2}\right)_{h}-\left(D_{x}^{2} \zeta^{j-1 / 2}, \zeta^{j-1 / 2}\right)_{h}\right. & \\
& \left.+\left|\zeta^{j+1 / 2}\right|_{h}^{2}+\left|\zeta^{j-1 / 2}\right|_{h}^{2}\right\}
\end{aligned}
$$

$$
\begin{aligned}
& +K \Delta t \sum_{j=1}^{n-1} \sum_{i=0}^{N-1} h_{i}^{2 k+4}\left\|D_{t}^{2} u^{j}\right\|_{H^{k+4}}^{2}\left(I_{i}\right) \\
& +\Delta t \sum_{j=1}^{N-1}\left\|e_{2}^{j}\right\|_{L^{2}(I)}^{2},
\end{aligned}
$$

where $\left\|e_{s}^{j}\right\|_{L^{2}(I)}=O\left(\Delta t^{2}\right)$ for $s=1,2, K$ is a generic constant and $\epsilon$ a constant that can be small enough.

Finally, by arguments similar to those of Section 4 we can show that

$$
\begin{aligned}
\Delta t & \sum_{j=1}^{n-1}\left(f\left(w^{j, 1 / 4}, D_{x} u^{j, 1 / 4}\right)-f\left(w^{j, 1 / 4}, D_{x} w^{j, 1 / 4}\right), \frac{\zeta^{j+1 / 2}-\zeta^{j-1 / 2}}{\Delta t}\right)_{h} \\
\leqslant & \frac{1}{\epsilon}\left[-\left(D_{x}^{2} \zeta^{n-1 / 2}, \zeta^{n-1 / 2}\right)_{h}+\left|\zeta^{n-1 / 2}\right|_{h}^{2}-\left(D_{x}^{2} \zeta^{1 / 2}, \zeta^{1 / 2}\right)_{h}+\left|\zeta^{1 / 2}\right|_{h}\right] \\
& +K \max _{0 \leqslant t^{j} \leqslant T} \sum_{j=0}^{N-1} h_{i}^{2 k+4}\left\|u^{j}\right\|_{H^{k+4}}+\max _{0 \leq t^{j} \leqslant T}\left\|e_{1}^{j}\right\|_{L^{2}(I)}^{2} \\
& +\frac{1}{\epsilon} \Delta t \sum_{j=1}^{n-1}\left[-\left(D_{x}^{2} \zeta^{j+1 / 2}, \zeta^{j+1 / 2}\right)_{h}+\left|\zeta^{j+1 / 2}\right|_{h}^{2}\right. \\
& +\Delta t \sum_{j=1}^{n-1}\left\|e_{3}^{j}\right\|_{H^{k+3}}^{2}(I) \\
& +K \Delta t \sum_{j=1}^{n-1} \sum_{i=0}^{N-1} h_{i}^{2 k+4}\left[\left\|u^{j}\right\|_{H^{k+3}}^{2}\left(_{\left(I_{i}\right)}^{2} \zeta^{j-1 / 2}, \zeta^{j-1 / 2}\right)_{h}+\left|\zeta^{j-1 / 2}\right|_{h}^{2}\right]
\end{aligned}
$$

where $e_{3}^{j}=O\left(\Delta t^{2}\right)$. From (5.6)-(5.8) and the discrete form of the Gronwall Lemma we derive in [6] the relation 


$$
\begin{aligned}
& \left|\partial_{t} \zeta^{n-1 / 2}\right|_{h}^{2}+\left|\zeta^{n-1 / 2}\right|_{h}^{2}-\left(D_{x}^{2} \zeta^{n-1 / 2}, \zeta^{n-1 / 2}\right)_{h} \\
& \leqslant C\left\{-\left(D_{x}^{2} \zeta^{1 / 2}, \zeta^{1 / 2}\right)_{h}+\left|\zeta^{1 / 2}\right|_{h}^{2}\left|\partial_{t} \zeta^{1 / 2}\right|_{h}^{2}\right\} \\
& +K \Delta t \sum_{j=1}^{n-1} \sum_{i=0}^{N-1} h_{i}^{2 k+4}\left[\left\|u^{j}\right\|_{H^{k+3}\left(I_{i}\right)}^{2}+\left\|D_{t}^{2} u^{j}\right\|_{H^{k+4}}^{2}\right] \\
& +C \Delta t \sum_{j=1}^{n-1}\left\{\left|\partial_{t}^{2} \eta^{j}\right|_{h}^{2}+\left|\eta^{j-1 / 2}\right|_{h}^{2}\right\}+\Delta t \sum_{j=1}^{n-1}\left[\left\|e_{2}^{j}\right\|_{L^{2}(I)}^{2}+\left\|e_{3}^{j}\right\|_{L^{2}(I)}^{2}\right] \\
& +K\left[\max _{0 \leqslant t^{j}<T} \sum_{i=0}^{N-1} h_{i}^{2 k+4}\left\|u^{j}\right\|_{H^{k+4}}^{2}\left(I_{i}\right)+\max _{0 \leqslant t^{j} \leqslant T}\left\|e_{1}^{j}\right\|_{L^{2}(I)}^{2}\right] .
\end{aligned}
$$

Finally from Lemma 1.4, 2.2 and inequality (5.9), we conclude that

$$
\begin{aligned}
\|\zeta\|_{L_{\Delta t}^{\infty}\left(0, T ; L^{\infty}\right)} \leqslant & C\left[\left\|\zeta^{1 / 2}\right\|_{H^{1}(I)}+\left\|\partial_{t} \zeta^{1 / 2}\right\|_{L^{2}(I)}\right] \\
& +K h^{k+2}\left[\|u\|_{\tilde{L}_{\Delta t}^{2}\left(0, T ; H^{k+3}(I)\right)}+\left\|D_{t}^{2} u\right\|_{\tilde{L}^{2}\left(0, T ; H^{k+4}(I)\right)}\right. \\
& \left.+\|u\|_{L_{\Delta t}^{\infty}\left(0, T ; H^{k+4}(I)\right)}\right] \\
& +c(u) \Delta t^{2},
\end{aligned}
$$

where $C$ and $K$ are generic constants independent of $u, h, \Delta t$ and $c(u)$ independent of $h, \Delta t$. From the results of Section 2 we easily see that

$$
\|\eta\|_{L_{\Delta t}^{\infty}\left(0, T ; L^{\infty}\right)} \leqslant C h^{k+2}\|u\|_{L_{\Delta t}^{\infty}\left(0, T ; H^{k+2}\right)} .
$$

Therefore, the inequalities (5.10) and (5.11) imply

$$
\left\|u-u_{h}\right\|_{L_{\Delta t}^{\infty}\left(0, T ; L^{\infty}\right)} \leqslant c(u)\left(h^{k+2}+(\Delta t)^{2}\right),
$$

provided

$$
\left\|\zeta^{1 / 2}\right\|_{H^{1}(I)}+\left\|\partial_{t} \zeta^{1 / 2}\right\|_{L^{2}(I)} \leqslant c h^{k+2}
$$

where $c(u)$ is independent of $h$ and $\Delta t$. This concludes the proof of Theorem 5.1.

It remains to discuss the choice of $u_{h}^{0}$ and $u_{h}^{1}$. We choose $u_{h}^{0} \equiv T_{h} u(x, 0)$ and $u_{h}^{1} \equiv T_{h} \tilde{u}$ where

$$
\widetilde{u} \equiv u(x, 0)+\Delta t D_{t} u(x, 0)+\frac{(\Delta t)^{2}}{2} D_{t}^{2} u(x, 0)+\frac{(\Delta t)^{3}}{6} D_{t}^{3} u(x, 0)
$$

the derivatives $D_{t}^{2} u$ and $D_{t}^{3} u$ are evaluated using the differential equation.

6. The Superconvergence Phenomenon. Consider the linear hyperbolic problem

$$
p(x, t) D_{t}^{2} u-D_{x}^{2} u=f(x, t), \quad(x, t) \in(0,1) \times(0, T),
$$

subject to initial conditions 


$$
u(x, 0)=\varphi_{1}(x), \quad D_{t} u(x, 0)=\varphi_{2}(x), \quad 0 \leqslant x \leqslant 1,
$$

and boundary conditions

$$
u(0, t)=0, \quad u(1, t)=0, \quad 0<t \leqslant T .
$$

Also, we assume for all $(x, t) \in[0,1] \times[0, T]$,

$$
0<m<p(x, t) \leqslant M, \quad 0<m \leqslant q(x, t) \leqslant M .
$$

Let $u_{h}$ denote the collocation on lines approximation defined from (3.5) and (3.6) where $p, q$ and $f$ are independent of $u$. Throughout we denote by $L \equiv p D_{t}^{2}-D_{x}^{2},\|u\|_{j, i} \equiv$ $\sup \left\{D_{x}^{\alpha} D_{t}^{\beta} u(x, t) \mid x \in I, \alpha \leqslant j, \beta \leqslant i\right\}$ and $x_{i-1 / 2} \equiv(i-1 / 2) h_{j}$. By Peano's Kernel Theorem [9] we obtain

$$
\begin{aligned}
L\left(u-T_{h} u\right) & \left(\zeta_{k j+i}, t\right) \\
= & \sum_{l=1}^{s-2}\left\{D_{x}^{k+l+1} D_{t}^{2} u\left(x_{j-1 / 2}\right) \psi_{l}\left(\rho_{i}\right)-D_{x}^{k+l+3} u\left(x_{j-y_{2}}\right) \psi_{l+2}^{\prime \prime}\left(\rho_{i}\right)\right\} h_{j}^{k+l+1} \\
& -D_{x}^{k+3} u\left(x_{j-1 / 2}\right) \psi_{2}^{\prime \prime}\left(\rho_{i}\right) h_{j}^{k+1}+O\left(h_{j}^{k+s}\left[\|u\|_{k+s+2,0}+\|u\|_{k+s+2,2}\right]\right),
\end{aligned}
$$

where

$$
\psi_{i}(x)=\frac{1}{(k+i+1) !} A_{i}(x) R_{k-2}(x)
$$

with $A_{i}$ a polynomial of degree $i-1$. In order to cancel the term of $h_{j}^{k+1}$ accuracy we make a correction to $T_{h} u$ defined locally by the following relations, $\delta_{0}(\cdot, t) \in$ $\mathbf{P}_{k+2, \Delta_{x}} \cap C^{1}$ with

$$
\begin{gathered}
h^{s-1} D_{x}^{2} \delta_{0}\left(\xi_{k j+i}, t\right)=D_{x}^{k+3} u\left(x_{j-y_{2}}\right) \psi_{2}^{\prime \prime}\left(\rho_{i}\right), \quad i=1, \ldots, k, j=0, \ldots, N-1, \\
\delta_{0}\left(x_{j}, t\right)=D_{x} \delta\left(x_{j}, t\right)=0, \quad j=0,1, \ldots, N .
\end{gathered}
$$

Now, in order to cancel the $h_{j}^{k+l+1}$ order terms we define a new correction in the following way: first we introduce the function

$$
v(y)= \begin{cases}0, & y \leqslant 0, \\ 3 y^{2}-2 y^{3}, & 0 \leqslant y \leqslant 1, \\ 1, & 1 \leqslant y,\end{cases}
$$

which obviously belongs to $C^{\mathrm{i}}$ and define for $x \in I_{j}$

$$
E_{j}(x, t) \equiv \lambda_{1, l} D_{x}^{k+l+1} D_{t}^{2} u\left(x_{j-1 / 2}\right) v\left(\frac{x-x_{j}}{h_{j}}\right)-\lambda_{2, l} D_{x}^{k+l+3} u\left(x_{j-1 / 2}\right) v\left(\frac{x-x_{j}}{h_{j}}\right),
$$

where $\lambda_{1, l} \equiv-\psi_{l}\left(\rho_{i}\right) / v^{\prime \prime}\left(\rho_{i}\right), \lambda_{2, l} \equiv-\psi_{l+2}^{\prime \prime}\left(\rho_{i}\right) / v^{\prime \prime}\left(\rho_{i}\right)$. Also, we define 


$$
\begin{aligned}
\delta_{l}(x, t) & \equiv \sum_{j=0}^{N-1} h_{j}^{l+3-s}\left\{E_{j}(x, t)-x E_{j}(1, t)\right\} \\
& =\sum_{j=0}^{N-1}\left\{\lambda_{1, l} D_{x}^{k+l+1} D_{t}^{2} u\left(x_{j-1 / 2}\right)-\lambda_{2, l} D_{x}^{k+l+3} u\left(x_{j-1 / 2}\right)\right\}\left(v\left(\frac{x-x_{j}}{h_{j}}\right)-x\right) .
\end{aligned}
$$

In [6] we show that the $\lambda_{\alpha, l}$ for $\alpha=1,2$ are well defined and easily obtain

$$
L(u-\bar{u})\left(\xi_{k j+i}, t\right)=O\left(h_{j}^{k+s}\left[\|u\|_{k+s+2,0}+\|u\|_{k+s+2,2}\right]\right),
$$

where

$$
\bar{u}=T_{h} u+h_{j}^{k+s} \sum_{l=0}^{s-2} \delta_{l} .
$$

THEOREM 6.1. Let $u$ denote the solution of the problem (6.1) to (6.4) such that $u \in L^{\infty}\left(0, T ; H^{k+s+4}\right), s \leqslant k$ and $u_{h}$ is the collocation on lines approximation of $u$ defined by (3.5), (3.6). Then the error of approximation at the nodes satisfies

$$
\begin{aligned}
\max _{j}\left\|\left(u-u_{h}\right)\left(x_{j}, \cdot\right)\right\|_{L^{\infty}(0, T)} \leqslant & C h^{k+s}\left[\|u\|_{k+s+2,0}+\|u\|_{k+s+2,2}\right] \\
& +C\left[\left\|D_{t}\left(u_{h}-\bar{u}\right)\right\|_{L^{2}(I)}(0)+\left\|u_{h}-\bar{u}\right\|_{H^{1}(I)}(0)\right],
\end{aligned}
$$

where $C$ is a constant independent of $u$ and $h$ and $s \leqslant k$.

Proof. We define

$$
\rho\left(\xi_{k j+i}, t\right) \equiv L\left(u_{h}-\bar{u}\right)\left(\xi_{k j+i}, t\right)
$$

where

$$
\left|\rho\left(\xi_{k j+i}, t\right)\right| \leqslant C h_{j}^{k+s}\left[\|u\|_{k+s+2,0}+\|u\|_{k+s+2,2}\right]
$$

and we form the relation

$$
\left(\rho, D_{t}\left(u_{h}-\bar{u}\right)\right)_{h}=\left(D_{t}^{2}\left(u_{h}-\bar{u}\right), D_{t}\left(u_{h}-\bar{u}\right)\right)_{h}-\left(D_{x}^{2}\left(u_{h}-\bar{u}\right), D_{t}\left(u_{h}-\bar{u}\right)\right)_{h} .
$$

We apply the elementary inequality $1 / 2 a^{2}+1 / 2 b^{2} \geqslant a b$ to obtain

$$
|\rho|_{h}^{2}+\left|D_{t}\left(u_{h}-\bar{u}\right)\right|_{h}^{2} \geqslant D_{t}\left|D_{t}\left(u_{h}-\bar{u}\right)\right|_{h}^{2}-D_{t}\left(D_{x}^{2}\left(u_{h}-\bar{u}\right), u_{h}-\bar{u}\right)_{h} .
$$

In the above inequality we add the inequality

$$
\frac{1}{2} D_{t}\left|u_{h}-\bar{u}\right|_{h}^{2} \leqslant \frac{1}{2}\left|D_{t}\left(u_{h}-\bar{u}\right)\right|_{h}^{2}+\frac{1}{2}\left|u_{h}-\bar{u}\right|_{h}^{2}
$$

to obtain

$$
\begin{aligned}
|\rho|_{h}^{2}+\mid u_{h}- & \left.\bar{u}\right|_{h} ^{2}+2\left|D_{t}\left(u_{h}-\bar{u}\right)\right|_{h}^{2} \\
& \geqslant D_{t}\left\{\left|u_{h}-\bar{u}\right|_{h}^{2}+\left|D_{t}\left(u_{h}-\bar{u}\right)\right|_{h}^{2}\right\}-D_{t}\left(D_{x}^{2}\left(u_{h}-\bar{u}\right), u_{h}-\bar{u}\right)_{h} .
\end{aligned}
$$

We integrate from 0 to $t$ and apply Gronwall's Lemma to get 


$$
\begin{aligned}
& C \int_{0}^{T}|\rho|_{h}^{2}(\tau) d \tau+\left|u_{h}-\bar{u}\right|_{h}^{2}(0)+\left|D_{t}\left(u_{h}-\bar{u}\right)\right|_{h}^{2}(0)-\left(D_{x}^{2}\left(u_{h}-\bar{u}\right), u_{h}-\bar{u}\right)_{h}(0) \\
& \quad \geqslant\left|u_{h}-\bar{u}\right|_{h}^{2}+\left|D_{t}\left(u_{h}-\bar{u}\right)\right|_{h}^{2}-\left(D_{x}^{2}\left(u_{h}-\bar{u}\right), u_{h}-\bar{u}\right)_{h} .
\end{aligned}
$$

It follows from Lemmas $1.3,1.4,1.5$ that

$$
\begin{array}{r}
C\left\{\max _{t}|\rho|_{h}+\left\|D_{t}\left(u_{h}-\bar{u}\right)\right\|_{L^{2}(I)}(0)+\left\|u_{h}-\bar{u}\right\|_{H^{1}(I)}(0)\right\} \\
\geqslant\left\|u_{h}-\bar{u}\right\|_{H^{1}(I)}+\left|D_{t}\left(u_{h}-\bar{u}\right)\right|_{h}^{2} .
\end{array}
$$

In particular, we have

$$
C\left\{\max _{t}|\rho|_{h}+\left\|D_{t}\left(u_{h}-\bar{u}\right)\right\|_{L^{2}(I)}(0)+\left\|u_{h}-\bar{u}\right\|_{H^{1}(I)}(0)\right\} \geqslant\left\|u_{h}-\bar{u}\right\|_{L^{\infty}(I)} .
$$

It is easy to see that

$$
\left|(u-\bar{u})\left(x_{i}, t\right)\right| \leqslant C h^{k+s}\left[\|u\|_{k+s-1,2}+\|u\|_{k+s+1,0}\right],
$$

where $h=\max _{j} h_{j}$. Consequently, we have

$$
\begin{aligned}
\max _{t} \max _{0 \leqslant j \leqslant N}\left|\left(u-u_{h}\right)\left(x_{j}, t\right)\right| \leqslant & C h^{k+s}\left[\|u\|_{k+s+2,0}+\|u\|_{k+s+2,2}\right] \\
& +C\left\{\left\|D_{t}\left(u_{h}-\bar{u}\right)\right\|_{L^{2}}(0)+\left\|u_{h}-\bar{u}\right\|_{H^{1}(I)}(0)\right\} .
\end{aligned}
$$

This completes the proof of Theorem 6.1.

Now, we consider the problem of choosing initial values, in order to obtain maximum accuracy. It is clear that the following

$$
u_{h}(x, 0)=T_{h} \varphi_{1}+h^{k+s} \delta_{0}(x, 0), \quad D_{t} u_{h}(x, 0)=T_{h} \varphi_{2}+h^{k+s} \delta_{0}(x, 0)
$$

yields $\left(u_{h}-\bar{u}\right)(0)=O\left(h^{k+s}\right)$ in $H^{1}$ norm, and $D_{t}\left(u_{h}-\bar{u}\right)(0)=O\left(h^{k+s}\right)$ in $L^{2}$ norm.

Department of Computer Sciences

Purdue University

West Lafayette, Indiana 47904

1. I. S. BEREZIN \& N. P. ŽIDKOV, Computing Methods, Vols. I, II, Fizmatgiz, Moscow, 1962; English transl., Addison-Wesley, Reading, Mass.; Pergamon Press, New York, 1965. MR 22 \#1 2685; 30 \#4372.

2. E. A. CODDINGTON, An Introduction to Ordinary Differential Equations, PrenticeHall, Englewood Cliffs, N.J., 1961. MR 23 \#A3869.

3. JIM DOUGLAS, JR. \& TODD DUPONT, “A finite element collocation method for quasilinear parabolic equations," Math. Comp., v. 27, 1973, pp. 17-28. MR 49 \#4266.

4. JIM DOUGLAS, JR. \& TODD DUPONT, "A super convergence result for the approximate solution of the heat equation by a collocation method," Mathematical Foundations of Finite Element Method with Applications to Partial Differential Equations (A. K. Aziz, Editor), Academic Press, New York, 1972.

5. JIM DOUGLAS, JR. \& TODD DUPONT, Collocation Methods for Parabolic Equations in a Single Space Variable (Based on C $C^{1}$-Piecewise-Polynomial Spaces), Springer Lecture Notes in Math., Vol. 385, Springer-Verlag, Berlin and New York, 1974. 
6. E. N. HOUSTIS, Finite Element Methods for Solving Initial/Boundary Value Problems, Doctoral thesis, Purdue University, 1974.

7. L. V. KANTOROVIČ, "Sur une méthode de resolution approchée d'equations différentielles aux derivées partielles," C. R. Acad. (Dokl.) Sci. URSS, v. 2, 1934, pp. 532-536. (Russian)

8. E. B. KARPILOVSKAJA, "Convergence of a collocation method for certain boundaryvalue problems of mathematical physics," Sibirsk. Mat. Ž., v. 4, 1963, pp. 632-640. (Russian) MR 27 \#6402.

9. M. H. SCHULTZ, Spline Analysis, Prentice-Hall, Englewood Cliffs, N.J., 1973. MR 50 \#1 5270.

10. Yu. P. YARTSEV, "Convergence of the collocation method on lines," Differencial'nye Uravnenija, v. 3, 1967, pp. 1606-1613 = Differential Equations, v. 3, 1967, pp. 838-842.

11. Yu. P. YARTSEV, "The method of line collocation," Differencial'nye Uravnenija, v. 4, 1968, pp. 925-932 = Differential Equations, v. 4, 1968, pp. 481-485. 